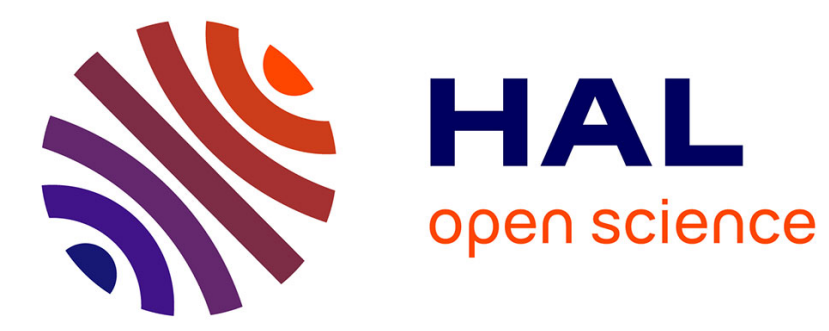

\title{
Étude des trajectoires d'apprentissage autodirigé : place de la planification, des intentions, du hasard. Peggy Candas
}

\section{To cite this version:}

Peggy Candas. Étude des trajectoires d'apprentissage autodirigé : place de la planification, des intentions, du hasard.. Carrefours de l'éducation, 2012, 2012/1 (33), pp.149-162. 10.3917/cdle.033.0149 . hal-00943525

\section{HAL Id: hal-00943525 \\ https://hal.science/hal-00943525}

Submitted on 7 Feb 2014

HAL is a multi-disciplinary open access archive for the deposit and dissemination of scientific research documents, whether they are published or not. The documents may come from teaching and research institutions in France or abroad, or from public or private research centers.
L'archive ouverte pluridisciplinaire HAL, est destinée au dépôt et à la diffusion de documents scientifiques de niveau recherche, publiés ou non, émanant des établissements d'enseignement et de recherche français ou étrangers, des laboratoires publics ou privés. 
Étude des trajectoires d'apprentissage autodirigé : place de la planification, des intentions, du hasard.

Peggy Candas

peggy.candas@unistra.fr

\section{État des lieux et objet de la recherche}

La société contemporaine envisage le citoyen comme individu capable de se former tout au long de la vie. En France comme en Europe, un courant de recherche, centré sur l'idée d'autoformation, s'est intéressé au rôle central de l'autonomie dans l'acte d'apprendre, d'abord chez l'adulte, pour s'étendre ensuite aux apprenants de tout âge (p. ex. Carré, 2005 ; Dumazedier, 2002 ; Little, 1991 ; Albero, 2003). Les auteurs de nombreux ouvrages et articles (p. ex. Carré, 1992 ; Carré et al, 1997 ; Courtois et Prévost, 1998 ; Foucher et Hrimech, 2000 ; Carré et Moisan, 2002) ont tenté d'opérationnaliser cette notion afin de donner les moyens aux acteurs de l'éducation et de la formation de promouvoir le développement de l'autonomie d'apprentissage chez les apprenants, pour ainsi permettre au plus grand nombre d'apprendre tout au long de la vie. La revue de la littérature montre que le terme « autonomie », que nous avons requalifié en « autonomie d'apprentissage », n'est pas défini précisément et que les conseils pratiques donnés aux éducateurs trouvent leur origine dans un autre courant de recherche, principalement nord-américain, celui de l'apprentissage autodirigé chez les adultes (cf. Tremblay et Eneau, 2006, p. 76-79), avec en particulier les travaux fondateurs de Knowles (1971) et Tough (1971). Holec, pionnier en France et auprès du Conseil de l'Europe, distingue l'autonomie, définie comme une capacité ou « une 'puissance de faire quelque chose', un 'pouvoir' », et l'auto-direction de l'apprentissage, qui est « un comportement, un 'faire quelque chose', le 'faire' étant ici l'utilisation de la capacité » (Holec, 1981, p. 21). Il établit ainsi clairement le lien entre autonomie et auto-direction de l'apprentissage, lien qui est très largement admis dans la littérature sur l'autonomie. Dans le champ de l'apprentissage autodirigé, la dimension organisationnelle reste la plus étudiée, et le consensus qui se dégage des nombreux articles publiés est que la planification est centrale dans le processus d'apprentissage autodirigé des adultes :

«In previous research in self-directed learning, Tough (1971), Peters and Gordon (1974), Penland (1977), and Hiemstra (1975) have reported or implied the existence of detailed preplanning of selfdirected learning by adults.

Specifically, Tough (1971, pp. 116-117) identified thirteen steps in planning that the learner "sometimes takes in a self-planned project." These steps include: identifying in detail what is to be learned, resources and methods for learning, time devoted to the effort, where to learn, pace of learning, deadline for completion, etc. » (Spear and Mocker, 1984, p. 2)

Or, dans le champ nord-américain de l'éducation des adultes, certains travaux viennent contredire ce principe maintes fois repris, en particulier ceux de Spear et Mocker (1984), dont les résultats sont détaillés dans plusieurs ouvrages ou articles de synthèse sur l'autonomie (Eneau, 2000, p. 41-45 ; Benson, 2001, p. 77-78 ; Tremblay, 2003, p. 150) : 
«Spear and Mocker's findings are of interest because they call into question the assumption that autonomous learning is necessarily planned learning. » (Benson, 2001, p. 78)

Dans Spear et Mocker (1984), une première analyse des données avait montré, que, contrairement à leurs attentes, leurs sujets adultes, qui avaient mené à bien un projet d'apprentissage autodirigé, n'avaient rien planifié de manière précise au début du projet. Les cas de planification étaient rares, et très vagues qui plus est. Le processus d'apprentissage paraissant néanmoins ordonné, délibéré et logique, les auteurs ont cherché à identifier les facteurs ayant structuré ces projets d'apprentissage autodirigé. La seconde analyse a montré que les adultes étudiés n'avaient pas essayé de trouver un grand nombre de ressources variées afin de choisir les mieux adaptées pour la réalisation de leur projet, mais avaient utilisé des ressources en nombre très limité, voire une ressource unique, disponible(s) dans leur environnement immédiat ou trouvée(s) par hasard. Il s'agissait toujours de l'une des premières ressources trouvées. En outre, tout ce qui était nécessaire au processus d'apprentissage (la structure, les méthodes, les ressources et les conditions d'apprentissage) avait le plus souvent été fourni ou dicté par les circonstances. Le processus d'apprentissage progressait, non pas de manière linéaire, mais plutôt d'un épisode d'apprentissage à un autre, et ce n'était que rétrospectivement qu'il donnait l'impression d'être linéaire et dirigé vers un objectif à long terme. De ces résultats est née l'expression « organizing circumstance » (dont deux traductions sont disponibles - « cadre organisateur » selon Bouchard, 1993 ; « circonstance organisante » selon Eneau, 2000) :

«(...) the researchers derived the concept of the Organizing Circumstance which postulates that selfdirected learners, rather than preplanning their learning projects, tend to select a course from limited alternatives which occur fortuitously within their environment, and which structures their learning projects. » (Spear et Mocker, 1984, p. 4)

Dans la même veine, Danis et Tremblay (1985) ont montré que si, chez les adultes autodidactes, "l'apprentissage est activé et contrôlé par les apprenants eux-mêmes » (p. 427), le processus d'apprentissage ne semble pas composé d'activités d'apprentissage se succédant dans un ordre séquentiel et unidirectionnel (vers le but fixé), en suivant des étapes prédéterminées. L'étude remet en cause le principe de linéarité, qui « trahit un point de vue centré sur l'enseignement ou sur l'apprentissage réalisé en milieu éducatif formel » (p. 435), mais n'a pas permis de définir le type de «contrôle » exercé par l'apprenant :

«[Self-taught adults] seem to exert control over their whole learning endeavor : Throughout their learning process, self-taught adults assume the monitoring of their own learning, even when consulting external agents or when participating in formal educational activities ${ }^{l}$. Little is known to date about the type of monitoring which is involved here. » (Danis et Tremblay, 1987, p. 6)

Cependant, cette recherche apporte de nouveaux éléments de connaissance quant aux modes et aux facteurs de structuration des trajectoires de ces apprenants autodirigés :

\footnotetext{
${ }^{1}$ Dans l'article de Danis et Tremblay (1985), les phrases en italique correspondent aux principes de I'apprentissage adulte que leur étude a confirmés.
} 
«On retrouve plutôt chez les autodidactes étudiés plusieurs processus d'apprentissage possibles, composés d'étapes ou de séquences multidirectionnelles. Un premier principe qui ressort ici est le suivant: les autodidactes procèdent de manière heuristique à l'intérieur d'une démarche qui s'organise autour d'intentions qui, à leur tour, se définissent et se précisent sans a priori. Un deuxième principe, en mettant en lumière l'impact des événements fortuits sur la démarche d'apprentissage, s'oppose également à la conception d'une démarche unique qui ne laisse pas suffisamment place au hasard ou à la découverte : les autodidactes saisissent toute occasion que le hasard peut leur offrir pour apprendre.» (Danis et Tremblay, 1985, p. 429)

Autrement dit, les autodidactes ne semblent se fixer que des «buts très larges et flexibles » (p. 430), rarement choisis au début du projet et qu'ils ajustent en cours de processus en fonction de leurs goûts et besoins personnels et en fonction des « circonstances qui se présentent à l'intérieur de leur domaine d'apprentissage » (p. 430).

Spear et Mocker (1984) et Danis et Tremblay (1985) aboutissent à une même définition des trajectoires d'apprentissage autodirigé comme n'étant ni linéaires, ni soigneusement planifiées en amont par l'apprenant, mais structurées au moins autant par les ressources disponibles dans l'environnement immédiat et par le hasard - ensemble de « circonstances organisantes » (Spear et Mocker, 1984) - que par les intentions très générales et flexibles de l'apprenant.

Les Centres de Ressources de Langues (CRL) de l'Université Louis Pasteur² (ULP), que fréquentent des étudiants spécialistes d'autres disciplines que les langues, ont été créés pour développer à la fois les compétences langagières et culturelles de ces étudiants dans une ou plusieurs langues étrangères et leur autonomie d'apprentissage. L'historique de la création de ces centres après plusieurs années de réflexion et d'expérimentation par une équipe de chercheurs et d'enseignants en sciences de l'éducation et en didactique des langues a été documenté et analysé ailleurs (Poteaux, 2010). Le projet pédagogique des CRL de l'ULP s'inscrit résolument dans une perspective de formation tout au long de la vie et les principes sous-jacents au dispositif, tel qu'il a été pensé et mis en œuvre, sont ceux mis en avant dans les courants de recherche sur l'autoformation et l'apprentissage autodirigé. Plus précisément, sept centres de ressources de langues remplacent les salles de classe traditionnelles; ils accueillent les étudiants avec leurs enseignants. Un grand nombre de ressources, très variées, sont disponibles et des enseignants sont toujours présents. Tous les étudiants de licence et master doivent valider des $\mathrm{ECTS}^{3}$ pour au moins une langue étrangère ${ }^{4}$. Le développement de l'autonomie d'apprentissage tout en apprenant une ou plusieurs langues étrangères est un objectif explicite: lors de fréquentes interactions (étudiants-enseignants, entre pairs), les étudiants sont encouragés à réfléchir de

\footnotetext{
${ }^{2} \mathrm{Au} 1^{\mathrm{er}}$ janvier 2009, les trois universités strasbourgeoises, l'Université Marc Bloch, l'Université Robert Schumann et l'Université Louis Pasteur, ont fusionné pour devenir l'Université de Strasbourg.

${ }^{3}$ Abréviation de European Credits Transfer System (Système européen de transfert et d'accumulation de crédits). Un crédit ECTS équivaut à 25 à 30 heures de travail étudiant.

${ }^{4}$ Trois ECTS par semestre du L1 au L3 de la licence et deux fois trois ECTS en master.
} 
manière critique à leur travail, à organiser leur parcours en se fixant des objectifs d'apprentissage, en choisissant des ressources adaptées, en définissant des modalités pratiques de travail, et à auto-évaluer les apprentissages ainsi réalisés (Holec, 1979). En première année de licence, ils sont en partie évalués sur leur capacité à analyser de manière critique leur parcours du semestre et à définir des priorités pour le module de langue du semestre suivant.

Une étude (Candas, 2005) préliminaire dans les CRL de l'ULP a montré d'importants décalages entre les pratiques attendues et les pratiques observées : en effet, des paramètres très différents, à la fois internes, comme l'humeur du moment ou les préférences personnelles, et externes, en particulier le hasard ou l'environnement immédiat, semblaient être en grande partie à l'origine des choix. Les convergences avec les résultats des études de Spear et Mocker (1984) et Danis et Tremblay (1985) dans des contextes pourtant très différents nous ont conduite à étudier (Candas, 2009) de manière compréhensive, les pratiques d'apprentissage autodirigé d'étudiants des CRL de l'ULP afin de déterminer comment ils s'y prennent pour tenter d'apprendre une langue étrangère dans un CRL lorsque leurs enseignants et l'institution leur demandent d'auto-diriger leur processus d'apprentissage, encouragés et accompagnés dans cette prise de responsabilité par les enseignants. Nous avons ensuite tenté de répondre à la question suivante: dans quelle mesure et selon quelles modalités les apprenants ont-ils planifié, dirigé ou organisé leur processus d'apprentissage?

\section{Méthodologie}

Choix du type de données recueillies

Nous souhaitions recueillir des données sur l'activité d'étudiants apprenant une langue étrangère dans un CRL de l'ULP, activité étant entendue au sens large de choix, actions, interactions, etc. Vermersch (2003) a montré que, lorsque le chercheur demande à un expert d'expliquer en détail comment il procède pour mener à bien une tâche dans son domaine d'expertise, la séquence d'actions que le spécialiste décrit est différente de celle qu'il réalise en situation effective. D'où notre décision d'écarter le discours comme source première de données et de choisir l'observation directe, cette dernière nous permettant d'obtenir des descriptions détaillées et situées de l'activité effective d'apprenants dans un CRL. Cependant, des tests préliminaires ont rapidement montré que l'observation directe ne permettait pas d'appréhender l'activité des apprenants dans son ensemble, une partie de l'activité n'étant pas visible. En outre, selon le modèle de l'activité d'Astolfi (2008), inspiré de Leontiev (1975), le sens qu'une personne attribue à ses actions est essentiel pour comprendre une succession d'événements donnés. La méthodologie choisie pour le recueil de données combine donc, pour chaque étudiant, l'observation de séances de travail en CRL et des entretiens directifs ayant lieu immédiatement après les séances observées.

Population étudiée 
Quatre enseignants de trois CRL différents ont accepté que nous recrutions des volontaires dans leurs groupes d'étudiants. La population ciblée était la suivante : des étudiants de licence, en première année (L1), dans leur second semestre de formation (S2), fréquentant un CRL pour un module d'anglais obligatoire. Nous avons fait l'hypothèse que ce semestre d'études pouvait être propice pour l'étude du développement de l'autonomie d'apprentissage puisque les étudiants avaient récemment quitté le lycée pour intégrer l'université. De plus, comme l'année L1 est considérée par les enseignants des CRL comme une période cruciale pour l'étudiant dans le processus de familiarisation et d'appropriation du dispositif d'apprentissage, ceux-ci concentrent une grande partie de leurs efforts en tant que personne-ressource au suivi des étudiants de première année. Aucun autre critère de sélection n'a été imposé. Huit étudiants - cinq femmes et trois hommes - de filières diverses ont participé à notre recherche. L'objectif de l'étude a été présenté en termes très généraux à tous les participants, enseignants et étudiants : après nous être présentée comme étudiante faisant une thèse, nous avons très brièvement expliqué que, dans la littérature, il y avait peu d'études décrivant la manière dont les étudiants travaillent dans les CRL et que le but de cette recherche était d'en apprendre davantage sans préjuger de ce que les étudiants devraient ou ne devraient pas faire.

\section{Données recueillies}

Pour chaque étudiant, quatre séances en CRL ont été observées. La durée des séances en CRL, en moyenne d'une heure et demie, a grandement varié selon les disponibilités des étudiants. L'étudiant était libre d'interrompre ou de prolonger la séance en CRL à sa convenance; toutefois, il lui avait été demandé de s'arrêter une demi-heure avant son départ pour que l'entretien puisse avoir lieu dans de bonnes conditions. Les observations ont eu pour objet l'activité de l'étudiant dans le CRL au sens large, c'est-à-dire pas seulement au sens de travail intellectuel, ainsi que l'activité de tout autre personne interagissant avec l'étudiant observé. Elles se sont accompagnées d'une prise de notes détaillées du chercheur, sans grille préalablement établie pour ne pas limiter l'observation à des catégories prédéterminées. Dans l'optique de l'entretien, les actions qui semblaient nécessiter une explicitation étaient immédiatement surlignées. L'ensemble des traces écrites produites par l'étudiant pendant la séance ont été photocopiées, ainsi que tous les documents de travail utilisés par l'étudiant qui pouvaient l'être.

Immédiatement après chaque séance en CRL, l'étudiant a rencontré le chercheur pour un entretien individuel dans une salle adjacente au CRL, en dehors de la présence de l'enseignant, de pairs ou de tout autre personne, afin qu'il puisse s'exprimer librement. Les entretiens, d'une durée très variable (entre dix et cinquante minutes), ont eu lieu en français et ont été enregistrés avec un dictaphone numérique après accord préalable de l'étudiant. Selon Vermersch (2003), les personnes à qui l'on demande de décrire l'activité qu'elles ont effectuée dans une situation singulière ont tendance à évoquer des classes d'événements (c'est-à-dire ce qu'elles font généralement dans une situation d'un type donné) plutôt que des événements situés (c'est-à-dire ce qu'elles ont fait dans une situation spécifique, dans un lieu et à un moment donnés). C'est donc le chercheur qui a mené l'entretien en 
s'appuyant sur ses notes, prises lors de l'observation, ainsi que sur les documents de travail utilisés et les éventuelles traces écrites produites par l'étudiant. Le chercheur a décrit ce qu'il venait d'observer durant la séance de manière chronologique et a encouragé l'étudiant à compléter les observations en recourant à des questions ouvertes pour obtenir des détails sur des actions spécifiques sans orienter les réponses. Le chercheur a privilégié les questions portant expressément sur ce qui avait été observé et a interrompu et réorienté l'étudiant quand le discours de celui-ci ne portait plus sur une action observée. À partir du fichier son, les entretiens ont été retranscrits verbatim.

\section{Analyse des données}

Pour chaque étudiant, nous avons obtenu une somme importante de données. Il s'avère difficile pour le chercheur d'appréhender l'ensemble de données constitué par les notes prises lors des quatre séances d'observation et les transcriptions intégrales des quatre entretiens d'explicitation s'y rapportant. Afin d'en faciliter la lecture et l'analyse, un tableau de synthèse a été élaboré pour chaque étudiant, regroupant les données relatives aux actions observées et au discours du sujet sur ces actions. Dans ce tableau, les quatre séances sont décrites successivement. Les données issues des observations et celles provenant des entretiens sont présentées dans deux colonnes différentes. Le discours de l'étudiant sur les actions apparaît sous forme de citations, en regard des actions en question. Les citations sont verbatim (non simplifiées ou réécrites, et parfois très longues) afin de présenter les données brutes et de ne pas introduire l'interprétation du chercheur à ce stade. Toutefois, lorsque la citation ne semblait pas claire, des éléments de contexte ont pu être précisés entre parenthèses dans les mots du chercheur.

Une méthodologie de traitement des données a été conçue. Après que les entretiens aient été transcrits, les idées trouvées dans la littérature dans divers champs de recherche théorique et pratique en biologie, neurosciences et sciences de l'éducation ont été confrontées avec les données disponibles pour chaque sujet de notre recherche. Notre but était d'identifier des éléments communs à la littérature et au corpus de données qui pourraient permettre de progresser dans la compréhension du processus d'apprentissage autodirigé. Quatre axes ont ainsi été retenus pour l'analyse qualitative de l'activité des étudiants: les pratiques d'exploration, les modalités de contrôle du processus d'apprentissage, la mise en place de routines et la réflexion sur celles-ci, et enfin la gestion des contraintes institutionnelles. Le traitement des données a consisté à repérer dans les tableaux de synthèse, c'est-à-dire par étudiant et par séance, de manière méthodique et exhaustive les passages correspondant aux quatre axes d'analyse. Quatre couleurs différentes ont été utilisées, une pour chaque axe d'analyse. L'analyse croisée des données d'observation et des données d'entretien a donné lieu à huit études de cas selon les quatre axes d'analyse. Une analyse comparative a ensuite été effectuée pour dégager des hypothèses sur les modes de structuration des trajectoires d'apprentissage autodirigé en milieu académique. 


\section{Résultats}

Nous présentons ici une partie des résultats obtenus, en lien avec le questionnement exposé plus haut. Lors des séances observées, aucun cas de paralysie (aucune action) ou d'errance (action sans intention sous-jacente, même vague) n'a été constaté : les sujets étudiés ont toujours choisi une ressource sur laquelle ils ont travaillé assez longtemps. La plupart des étudiants ont pris très peu de temps pour choisir une ressource, que ce soit en début de séance ou plus tard, après avoir exploré une toute petite partie des ressources disponibles dans le centre (par exemple, en consultant la liste des films dans le catalogue de ressources; en balayant du regard les revues exposées sur le présentoir). Concernant le choix d'une ressource, les étudiants n'ont cité que très rarement un objectif d'apprentissage : souvent ils ne savaient pas ou n'ont pas pu dire pourquoi ils avaient choisi de travailler avec telle ou telle ressource, ou, s'ils ont mentionné un but, celui-ci était très général (par exemple, apprendre plus de vocabulaire, mieux comprendre l'anglais parlé). Ils ont souvent dit avoir choisi la ressource parce qu'elle leur plaisait. Ainsi, l'étudiante Et10 jouait fréquemment en français à un jeu de société que le centre mettait à disposition en anglais et elle a voulu essayer cette version du jeu. Lorsque le chercheur a insisté, certains étudiants ont justifié leur choix en utilisant un ensemble de critères plus ou moins vagues en rapport avec l'idée qu'ils se faisaient de la pertinence linguistique d'une ressource (parce qu'elle était familière ou d'après ce que des enseignants d'anglais en avaient dit) et de la manière d'apprendre une langue étrangère, en rapport aussi avec des expériences d'apprentissage passées et d'autres paramètres plus terre-à-terre comme ce qu'ils avaient envie de faire ce jour-là et leur degré de fatigue. Cependant, ces critères ne semblaient pas avoir été mobilisés consciemment et rationnellement au moment précis de faire le choix. Ce n'est qu'après avoir travaillé sur une ressource assez longuement (souvent une séance complète d'une heure trente) que des étudiants ont dit vouloir continuer à travailler avec la même ressource ou une ressource du même type ultérieurement : par exemple, l'étudiante Et10, après avoir fini de regarder en anglais un film d'animation qu'elle avait déjà vu de nombreuses fois en français et connaissait presque par cœur, a déclaré que regarder une vidéo que l'on connaissait bien était un exercice plus utile à cause du travail que cela lui avait permis de faire, à savoir comparer les jeux de mots dans les deux langues. Le nombre limité de séances observées ne nous a pas permis de vérifier si, dans de tels cas, des ressources du même type étaient effectivement utilisées par la suite, sauf dans l'étude de cas de l'étudiant Et9, qui s'est servi du même ouvrage d'anglais scientifique avec cassettes audio lors de trois séances différentes parce qu'il sentait que cela lui permettait de progresser dans ses compétences d'écoute et dans la compréhension du vocabulaire scientifique.

Dans notre étude, nous avons également analysé comment les étudiants ont utilisé les ressources choisies. Carette et Holec (1995) distinguent les «matériels pré-adaptés » avec appareil didactique imposé et les «matériels adaptables» qui offrent une plus grande liberté d'utilisation (p. 87). Pour la première catégorie, la comparaison des différentes manières d'exploiter une ressource d'un même type s'est avérée riche d'enseignements. Prenons l'exemple d'une dictée sur cédérom : la tâche de l'apprenant consiste à transcrire 
verbatim phrase après phrase un enregistrement audio, le logiciel mettant une aide à disposition, à la demande de l'utilisateur, en donnant soit le sens de tel ou tel mot recherché (la fonction 'dictionnaire'), soit la bonne réponse pour ce mot. L'étudiante Et8, pour ne pas passer trop de temps sur chaque phrase, a développé une méthode où elle écoute une phrase un certain nombre de fois, fixe et limité, utilisant la fonction 'dictionnaire' pour les mots non trouvés sans s'arrêter, avant de demander la bonne réponse pour chacun d'entre eux. En revanche, les étudiants Et10 et Et9 écoutent chaque phrase un très grand nombre de fois, puis utilisent la fonction 'dictionnaire' pour un seul mot non trouvé avant de réécouter la phrase de nombreuses fois, procèdent ainsi avec le mot suivant et ainsi de suite, ne demandant au logiciel la bonne réponse pour un mot qu'après avoir épuisé toutes les autres possibilités ; ils trouvent cette manière de faire plus efficace pour progresser. L'étudiante Et8 effectue une série d'actions presque automatiques sans réflexion sur l'intérêt de sa manière de procéder en termes d'apprentissage, contrairement aux deux autres étudiants. Concernant la deuxième catégorie de ressources, l'étude des tâches effectuées avec les matériels adaptables a révélé des influences multiples, parmi lesquelles les modalités d'évaluation jouaient un rôle majeur (les étudiants devaient constituer un dossier comprenant tous leurs brouillons, notes et productions écrites et le montrer à l'enseignant-évaluateur), ainsi que les recommandations générales des enseignants et l'expérience scolaire des étudiants qui les ont toutes deux conduits à considérer certains types de tâches comme essentiels (noter les mots inconnus et leur traduction en français ; faire un résumé quelle que soit la ressource). Quand l'intervieweur a demandé aux étudiants Et5 et Et4 pourquoi il ou elle avait décidé de faire un résumé de l'article lu ou du film vu, le premier a répondu que, comme cela, il aurait fait quelque chose, alors que l'étudiante Et4 a dit que c'était pour elle, avant de se corriger et d'admettre qu'elle ne savait pas, pour finalement décrire cette tâche comme une habitude (à noter qu'au début de l'entretien suivant, elle a spontanément ré-abordé la question et avoué qu'après réflexion, elle pensait plutôt qu'elle faisait des résumés parce que c'était une contrainte pour la validation du module d'anglais). L'ensemble des données montrent très peu de variété quant aux tâches effectuées à partir des matériels adaptables ; en outre, les choix ainsi faits sont très rapidement décidés et semblent en quelque sorte automatiques. Cependant, nos données incluent deux cas très nets où la simple description de ses actions faite par l'étudiant au chercheur pendant l'entretien a conduit l'apprenant à reconsidérer sa manière de travailler de manière critique en fonction d'un objectif explicité à voix haute : ainsi, l'étudiante Et6 mettait systématiquement les sous-titres en anglais quand elle regardait des vidéos, mais, après que l'intervieweur lui ait demandé d'expliciter ce choix pour la séance sur laquelle l'entretien portait, l'étudiante a fini par dire que, même si elle n'y avait jamais pensé, il serait intéressant d'essayer sans les sous-titres pour améliorer ses compétences d'écoute.

\section{Discussion}

Notre étude des pratiques observées montre que les trajectoires d'apprentissage autodirigé des étudiants sont structurées par des buts très larges et flexibles, par une sorte d'« intentionnalité flottante et transversale » (Berten, 1999, p. 43) qui intègre aussi ce que 
les apprenants aiment et ressentent à un moment donné, confirmant l'importance de la dimension affective dans le processus d'apprentissage (Berthoz, 2003 ; Candas et Eneau, 2010 ; Damasio, 1995). Elles sont également structurées par des ressources découvertes par hasard et/ou aisément accessibles dans l'environnement immédiat des apprenants («the organizing circumstance » de Spear et Mocker, 1984). Pour faire référence à la façon dont les apprenants contrôlent ou organisent leur processus d'apprentissage, nous avons choisi de parler de pilotage flou : ce qui prime pour l'apprenant, c'est d'être dans une dynamique d'action et toute modification de la trajectoire d'apprentissage ou des modalités de travail est décidée chemin faisant, l'ensemble n'ayant pas été méticuleusement planifié en amont. Nos résultats vont dans le même sens que ceux obtenus par Danis et Tremblay (1985) et Spear et Mocker (1984) dans leurs études des projets autodirigés d'adultes. Ils contredisent l'idée dominante dans les courants de recherche sur l'autoformation et l'apprentissage autodirigé selon laquelle l'apprentissage autodirigé est nécessairement planifié. En outre, dans notre étude comme dans celle de Danis et Tremblay (1985), les apprenants évaluent un épisode d'apprentissage d'après les résultats pratiques obtenus ou d'après le sentiment d'avoir progressé ; ils ne cherchent pas de rétroaction de type formel et/ou institutionnel. L'ensemble de ces résultats nous conduisent à réfuter l'hypothèse selon laquelle le degré d'autonomie d'un apprenant serait en rapport avec ses capacités à organiser de manière rationnelle son processus d'apprentissage. Le contexte universitaire, avec ses multiples contraintes institutionnelles, induit bien entendu des biais : ainsi, les sujets de l'étude ont reconnu travailler principalement pour valider leurs ECTS de langue, même s'ils ont tous affirmé qu'ils aimaient l'anglais ou trouvaient cette langue utile, et aucun n'a évoqué de projet personnel précis nécessitant la maîtrise de cette langue. Ces étudiants étaient-ils tous très peu autonomes dans l'apprentissage des langues étrangères? Nous postulons qu'ils l'étaient à des degrés divers. Sur bien des points, le portrait de l'étudiant autodirigé qui se dégage de notre étude rejoint celui de l'adulte autodirigé issu des travaux de Tremblay (2003) qui a regroupé ses compétences autour des cinq notions entre parenthèses : l'adulte autodirigé est capable de transcender son expérience d'apprentissage et de comprendre le processus d'apprentissage (méta-apprentissage); il s'engage dans une démarche heuristique malgré toutes les incertitudes qui pourraient le paralyser, sait saisir toute occasion d'apprendre que le hasard place sur sa route (stochastique); ses intentions et son projet s'ajustent au gré de ses désirs, des circonstances et des ressources présentes dans son environnement (cadre organisateur); il évolue dans l'action et en fonction de l'expérience antérieure (praxis) et réussit à se constituer tout un réseau de ressources, matérielles et humaines (réseautage) pour réaliser un apprentissage.

Des différences notables entre les étudiants apparaissent surtout dans la manière de travailler avec les ressources choisies, en particulier avec les matériels pré-adaptés : un appareil didactique est fourni, ce qui n'empêche pas l'apprenant d'en faire l'utilisation qu'il souhaite. Notre recherche suggère que l'étude de ces usages ou modes d'appropriation donne des indications sur la capacité d'auto-direction de l'apprenant, et donc peut-être aussi sur son degré d'autonomie dans l'apprentissage d'une langue étrangère. Les matériels adaptables, quand ils sont sans consigne aucune, conduisent les 
étudiants à se rabattre sur les tâches connues (habitudes scolaires) ou celles qu'ils perçoivent comme étant valorisées par les enseignants. Globalement, les contraintes institutionnelles et les routines scolaires semblent laisser peu de place à la prise de distance critique nécessaire pour effectuer des choix informés, les étudiants étant assez logiquement prêts à faire tout ce qui leur apparaît comme étant requis pour l'obtention du diplôme. Néanmoins, deux études de cas dans notre recherche montrent que la simple verbalisation de son activité peut conduire un apprenant à réévaluer ses actions passées et à envisager une alternative peut-être plus efficace. Ce résultat, inattendu et obtenu grâce à la méthodologie de récolte des données, peut avoir des conséquences importantes sur la nature de l'intervention de l'enseignant dans les dispositifs à visée autonomisante : plutôt que d'encourager et d'entraîner les apprenants à déterminer des objectifs et des méthodes d'apprentissage, il pourrait s'avérer utile de se faire le simple miroir de leurs actions, de leur renvoyer l'image de ce qu'ils font et de leur donner l'occasion d'expliciter leur activité et leurs choix, dans la perspective d'une prise de conscience génératrice d'évolutions et de changements. Cette technique d'entretien rappelle bien évidemment l'entretien de conseil avec son protagoniste, le conseiller, dont le rôle a été théorisé par Gremmo (1995). Le rôle de l'enseignant pourrait s'en trouver profondément modifié ; le caractère primordial de son intervention pour déclencher une prise de distance critique est réaffirmé. Notre recherche ouvre ainsi des perspectives pour une meilleure connaissance des processus à l'œuvre dans les pratiques d'apprentissage autodirigé en formation initiale à l'université, et, par conséquent, pour la conception de dispositifs ad hoc.

\section{Conclusion}

Spear et Mocker (1984) et Danis et Tremblay (1985) semblent avoir ouvert de nouvelles pistes pour la compréhension du processus d'apprentissage autodirigé chez les adultes ; ces travaux n'ont à notre connaissance pas donné lieu à une remise en cause du modèle véhiculé par le courant de recherche dominant. Les résultats de notre étude sur les trajectoires d'apprentissage autodirigé d'étudiants montrent des similarités importantes : à la différence des (par)cours soigneusement planifiés par l'enseignant pour sa classe, les trajectoires d'apprentissage autodirigé sont le fruit de l'influence combinée de l'environnement immédiat, du hasard et des intentions plutôt vagues de l'apprenant. En relativisant l'importance de la planification, l'énorme responsabilité pesant sur les épaules de l'apprenant autodirigé se trouve allégée et les compétences indispensables pour entreprendre et réussir des apprentissages autodirigés ne sont plus les compétences organisationnelles de celui qui s'enseigne à lui-même. Nous constatons que même dans la conception de dispositifs innovants, le modèle transmissif dominant a été déterminant puisque les tâches habituelles de l'enseignant ont été dévolues à l'apprenant. Il est peutêtre temps de reconsidérer ce modèle et de redonner sa place à l'aléatoire, qui est au cœur du développement des systèmes vivants ${ }^{5}$.

\footnotetext{
${ }^{5}$ Cf. Linard (1996, p. 84) sur tout système vivant d'après le modèle connexionniste : « c'est son organisation structurelle interne qui produit, sélectionne et fait "émerger » automatiquement des configurations à
} 


\section{Peggy Candas, Université de Strasbourg, Strasbourg}

Laboratoire Interuniversitaire des Sciences de l'Éducation et de la Communication (LISEC)

\section{Références bibliographiques :}

Albero B. (dir.) (2003). Autoformation et enseignement supérieur. Paris : Hermès.

Astolfi J.-P. (2008). La Saveur des Savoirs : Disciplines et plaisir d'apprendre. Paris : ESF.

Benson P. (2001). Teaching and researching autonomy in language learning. Harlow : Longman/Pearson Education.

Berten A. (1999). Dispositif, médiation, créativité : petite généalogie. Hermès, n²5, p. 33-47.

Berthoz A. (2003). La Décision. Paris : Odile Jacob.

Bouchard P. (1993). Pourquoi apprendre seul? Les déterminants du choix éducatif chez des professionnels autodidactes. Thèse de doctorat, Université de Montréal.

Candas P. (2005). Les usages des étudiants dans les centres de ressources de langues de l'Université Louis

Pasteur: construction raisonnée d'un parcours ou itinéraire fortuit? Communication lors du Troisième

Colloque Mondial sur l'Autoformation («Rencontres entre les cultures et les pratiques d'apprentissage formelles, informelles, non formelles »), 23-25 novembre, Marrakech, Maroc.

Candas P. (2009). Analyse de pratiques d'étudiants dans un Centre de Ressources de Langues : indicateurs d'autonomie dans l'apprentissage. Thèse de doctorat en sciences de l'éducation, Université de Strasbourg.

Candas P., Eneau J. (2010). Autonomie de l'apprenant et dimensions affectives de l'autoformation. In B.

Albero et N. Poteaux (dir.) Enjeux et dilemmes de l'autonomie. Une expérience d'autoformation à l'université. Étude de cas. Paris : Les Éditions de la Fondation Maison des Sciences de l'Homme, p. 141-167. Carette E., Holec H. (1995). Quels matériels pour les centres de ressources? Mélanges Pédagogiques, $\mathrm{n}^{\circ} 22$, p. 85-94.

Carré P. (1992). L'autoformation dans la formation professionnelle. Paris : La Documentation Française.

Carré P. (2005). L'apprenance : Vers un nouveau rapport au savoir. Paris : Dunod.

Carré P., Moisan A. (dir.) (2002). La formation autodirigée: Aspects psychologiques et pédagogiques. Paris : L'Harmattan.

Carré P., Moisan A., Poisson D. (1997). L'autoformation: Psychopédagogie, ingénierie, sociologie. Paris : Presses Universitaires de France.

Courtois B., Prévost H. (dir.) (1998). Autonomie et formation au cours de la vie. Lyon : Chronique Sociale. Damasio A. (1995). L'erreur de Descartes : La raison des émotions. Paris : Odile Jacob.

Danis C., Tremblay N. (1985). Principes d'apprentissage des adultes et autodidaxie. Revue des Sciences de l'Éducation, vol. 11, n³, p. 421-440.

Danis C., Tremblay N. (1987). Propositions regarding autodidactic learning and their implications for teaching. Lifelong Learning, vol. 10, nº 7, p. 4-7.

Dumazedier J. (2002). Penser l'autoformation: Société d'aujourd'hui et pratiques d'autoformation. Lyon : Chronique Sociale.

Eneau J. (2000). Facteurs individuels et organisationnels d'influence de l'autoformation. Examen de synthèse de doctorat, Université de Montréal.

Foucher R., Hrimech M. (dir.) (2000). L'autoformation dans l'enseignement supérieur: Apports européens et nord-américains pour l'an 2000. Montréal : Éditions Nouvelles AMS.

Gremmo M.-J. (1995). Conseiller n'est pas enseigner: le rôle du conseiller dans l'entretien de conseil. Mélanges Pédagogiques, $\mathrm{n}^{\circ} 22$, p. 33-61.

Hiemstra R. (1975). The older adult and learning. Lincoln : University of Nebraska.

Holec H. (1979). Autonomie et apprentissage des langues étrangères. Strasbourg : Éditions du Conseil de l'Europe.

Holec H. (1981) À propos de l'autonomie - Quelques éléments de réflexion. Études de Linguistique Appliquée, $\mathrm{n}^{\circ} 41, \mathrm{p}$. 7-23.

Knowles M. (1971). The Modern Practice of Adult Education: Andragogy vs Pedagogy. New York: Association Press.

partir des circonstances aléatoires, au fil de l'historique du couplage système-milieu, et à partir d'une masse indéfinie de possibles (Varela, 1989) ». 
Leontiev A. (1975). Activity, Consciousness, Personality. Moscou : Progress Editions.

Linard M. (1996). Des machines et des hommes: Apprendre avec les nouvelles technologies. Paris: L'Harmattan.

Little D. (1991). Learner Autonomy - 1 : Definitions, Issues and Problems. Dublin : Authentik.

Penland P. (1977). Self-planned learning in America : Final report. Pittsburgh : University of Pittsburgh.

Peters J., Gordon S. (1974). Adult learning projects : a study of adult learning in urban and rural Tennessee. Knoxville : Tennessee University.

Poteaux N. (2010). Histoire d'une innovation et trajectoire d'acteurs. In B. Albero, N. Poteaux (dir.) Enjeux et dilemmes de l'autonomie. Une expérience d'autoformation à l'université. Étude de cas. Paris : Les Éditions de la Fondation Maison des Sciences de 1'Homme, p. 41-65.

Spear G., Mocker D. (1984). The organizing circumstance : environmental determinants in self-directed learning. Adult Education Quarterly, vol. 35, n¹, p. 52-77.

Tough A. (1971). The adult's learning project : A fresh approach to theory and practice in adult learning. Toronto : The Ontario Institute for Studies in Education.

Tremblay N. (2003). L'autoformation: pour apprendre autrement. Montréal : Presses Universitaires de Montréal.

Tremblay N., Eneau J. (2006). Sujet(s), société(s) et autoformation : regards croisés du Québec et de France. Éducation Permanente, vol. 3, n¹68, p. 75-88.

Varela F. (1989). Connaître. Les sciences cognitives, tendances et perspectives. Paris : Éditions du Seuil. Vermersch P. (2003). L'entretien d'explicitation. Issy-les-Moulineaux : ESF. 